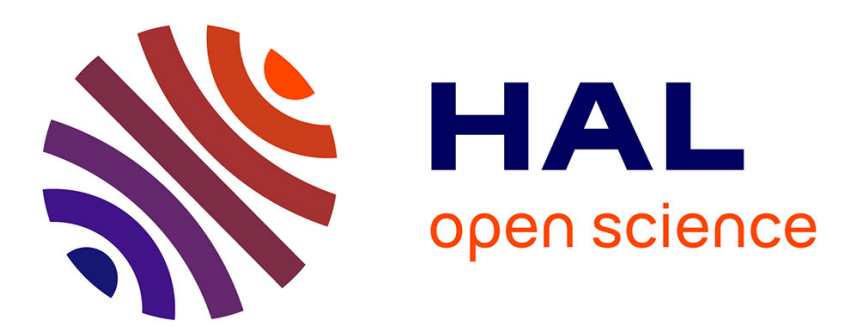

\title{
Properties of the pressure field in highly nonlinear free surface flows with critical jet
}

\author{
Yves-Marie Scolan, Stéphane Etienne
}

\section{To cite this version:}

Yves-Marie Scolan, Stéphane Etienne. Properties of the pressure field in highly nonlinear free surface flows with critical jet. Journal of Engineering Mathematics, 2021, 128 (1), 10.1007/s10665-021-101164. hal-03242648

\section{HAL Id: hal-03242648 \\ https://hal-ensta-bretagne.archives-ouvertes.fr/hal-03242648}

Submitted on 1 Jun 2021

HAL is a multi-disciplinary open access archive for the deposit and dissemination of scientific research documents, whether they are published or not. The documents may come from teaching and research institutions in France or abroad, or from public or private research centers.
L'archive ouverte pluridisciplinaire $\mathbf{H A L}$, est destinée au dépôt et à la diffusion de documents scientifiques de niveau recherche, publiés ou non, émanant des établissements d'enseignement et de recherche français ou étrangers, des laboratoires publics ou privés. 


\title{
Properties of the pressure field in highly nonlinear free surface flows with critical jet.
}

\author{
Yves-Marie SCOLAN ${ }^{(1)}$, Stéphane ETIENNE(2)
}

(1) ENSTA Bretagne IRDL UMR 6027, Brest, France

(2) Ecole Polytechnique de Montréal, Canada ${ }^{1}$

\begin{abstract}
In this note we examine fluid violent kinematics in a plunging breaker. The fluid motion is computed in the frame of the potential flow theory. The fluid kinematics is basically generated by forcing the motion of a rectangular tank, starting from rest. When a sufficient level of energy is injected in the fluid, the free surface has highly nonlinear behavior, here a plunging breaker. In the vicinity of the main tip crest, a sharp corner (cusp) appears along the surface of the barrel. The appearance of this critical jet is described and discussed in terms of the spatial and temporal variations of the pressure field. In the present case a local pressure maximum is captured that follows a continuous decreasing pressure gradient in a region of positive Gaussian curvature of the pressure. It is also shown that, at the free surface before the appearance of the critical jet, there is a strong correlation between the change of sign of the Gaussian curvature of the pressure on the one hand and the radius of curvature of the free surface profile on the other hand.
\end{abstract}

Keywords: Two-dimensional potential flow, plunging breaker, critical jet, pressure field.

\section{Introduction}

The circumstances under which a critical jet may appear on a free surface are hardly documented in the literature. Longuet-Higgins is certainly the main contributor to the analysis of nonlinear free surface flow in potential theory in all its aspects; particularly those of interest to us in this note. In particular in Longuet-Higgins (1980a), it is established that the spatial variations of the pressure provide much information about the appearance of sharp corners (cusps) at the free surface. By using the terminology of Longuet-Higgins, this sudden growth of the free surface is called a critical jet. For that the author examines the singularities of the pressure field. In order to capture such singularities, one has to compute the pressure gradient, the mean curvature (identical to the Laplacian) and the Gaussian curvature. The former is always negative for potential flows, the latter is either negative (saddle points in hyperbolic region) or positive (local maximum in elliptic region). The gaussian curvature is considered as the product of the principal curvatures, that is to say the inverse of the product of local radii of curvature.

Examples of the development of sharp corner on a free surface is theoretically studied in subsequent papers (see Longuet-Higgins, 1980b). It is shown that by continuing the

Email address: yves-marie.scolan@ensta-bretagne.fr, stephane.etienne@polymtl.ca (Yves-Marie SCOLAN $^{(1)}$, Stéphane ETIENNE ${ }^{(2)}$

(1) ENSTA Bretagne IRDL UMR 6027, Brest, France

(2) Ecole Polytechnique de Montréal, Canada) 
pressure field outside the fluid domain, local asymptotic solutions are provided and sharp corners along the zero pressure line (free surface) can be captured. Other theoretical solutions are also exhibited in Longuet-Higgins (1993). Critical jets are described in Longuet-Higgins and Dommermuth (2001b). In this article a small size jet is observed (in Fig. 6) however without giving any quantitative value of the local acceleration. The question of finite-time singular behavior is posed by Longuet-Higgins (1993). The power law is proposed and it is shown that acceleration behaves as $\left(t-t_{o}\right)^{-\frac{4}{3}}$ where $t_{o}$ is somewhat arbitrary. The notion of "inertial shock" is introduced and associated to singular values of the velocity, pressure and acceleration while fluid displacements remain finite. Such flows are observed experimentally and compare well with theoretical results like in Zeff et al (2000) or LonguetHiggins and Dommermuth (2001b). The so-called flip-through (see Cooker and Peregrine, 1990) phenomenon belongs to this type of accelerated flow as well. Cooker (2001) and Cooker (2010) exhibits local solutions of the pressure starting with a local free surface deformation like a semicircular trench. It is shown that great pressure gradient and acceleration are expected below the free surface just below the initial crater. This has common features with the bazooka effect described in Longuet-Higgins (2001a).

If theoretically it is shown that cusps or sharp corners can appear on a free surface, we still need to better examine how such critical jets are associated with high kinematics. Grilli and Subramanya (1996) observe in their computations the appearance of a critical jet in the close vicinity of the tip of a plunging breaker. It seems that this occurrence is associated with a rapid fluid motion. In their case the fluid motion follows from an impulsive motion of a wave maker. Indeed we can expect that a large amount of energy must be stored by the fluid so that critical jets can appear. That is a conjecture but numerical experiments in a sloshing tank confirm that (see Scolan et al, 2016; Scolan, 2018).

In the present work, as in Longuet-Higgins (1980a) and Cooker (2001), we analyse the pressure field before the appearance of a critical jet. Here, we are interested in the combination of both the positive Gaussian curvature of the pressure and the zeros of pressure gradient. Those quantities are computed for some "exotic" free surface flows that are simulated in a sloshing tank. We focus on one configuration where a standard plunging breaker develops as a result of the forced motion of the tank. Then, as the crest starts overturning a sudden secondary jet appears in the close vicinity of the main crest tip. From a parametrical study it is possible to tune some parameters of the forced tank motion in order to make the critical jet more prominent. That is the objective of this note to describe the spatialtemporal variations of the scalar or vector fields (pressure, acceleration,...) before and after the critical jet appears.

This note is decomposed as follows. Section 2 recalls the main equations of the model. The expression of the pressure and its derivatives are described as well. In section 3, the conditions that lead to the studied case are detailed. Then the spatial-temporal variations of scalar or vector fields (pressure, curvatures, acceleration,...) are discussed. The conclusion draws the perspectives of future works.

\section{Governing equations}

The numerical model is detailed in Scolan (2010). It follows from the pioneering numerical developments proposed by Tuck (1998). The boundary value problem is here formulated for the velocity potential $\Phi$ that describes the flow in the moving tank as illustrated in figure (1) We consider the forced motion of a two-dimensional tank. Three degrees of freedom (dof) can be considered: two translational motions and one rotational motion. The velocity 
potential $\Phi$ verifies a boundary value problem which reads

$$
\begin{cases}\Delta \Phi=0 & \text { in the fluid domain } \\ \Phi_{, t}+\frac{1}{2} \vec{\nabla}^{2} \Phi+g(\vec{Y} \cdot \overrightarrow{E P}-h)=0 & P \text { on the free surface } \\ \frac{d}{d t} \overrightarrow{E P}=\vec{\nabla} \Phi & P \text { on the free surface } \\ \Phi_{, n}=\frac{d}{d t} \vec{M} \cdot \vec{n} & M \text { on the wall of the tank }\end{cases}
$$

where $\vec{n}$ is the unit normal vector on any considered surface pointing outside the fluid.

The velocity potential $\Phi$ is decomposed classicaly into two contributions. One is known as the Stokes-Joukowski velocity potential $\phi$ and it accounts for the forced motion of the tank (see Faltinsen et al, 2000). The other contribution $\varphi$ captures the fluid motion in the moving tank. The former velocity potential reads

$$
\phi=\vec{v}_{A} \overrightarrow{A P}
$$

where $\vec{v}_{A}$ is the translational velocity of the point $A$. No rotational forced motion is considered here but the full problem with both translational and rotational forced motions is described in Scolan and Brosset (2017). The boundary conditions for $\varphi$ are a simple homogeneous Neumann condition on the tank walls and it is completed with the dynamic boundary condition on the free surface. Two techniques are combined in order to have a robust and fast computation of nonlinear free surface potential flows. The equations are two-dimensional which makes it possible to use a conformal mapping of the physical computational domain. For example a Schwartz-Christoffel transformation (see Spiegel, 1999, p. 206) leads to the following analytic function

$$
w=-\cos \frac{\pi z}{L}
$$

This transformation simply turns the inner domain of a semi-infinite strip (width $L$ ) defined in the upper physical $z$-plane, into a half upper $w$-plane. The origin $O$ of the coordinate system in the physical plane is always located at the bottom left corner. The images of the vertical walls and horizontal bottom are now located along the real axis of $w$-plane. The fluid motion is described with the velocity potential $\varphi$. It is enforced through a dsitribution of sources. Instead of a distribution on the actual physical boundaries, it is preferred a desingularized technique. The advantage (among some others) is to avoid the additional work induced by the discretization of an integral equation. Convergence tests from Cao et al (1991) show how to choose the desingularizing distance for the present applications. This choice follows from considerations about the conservations of mass and energy which have to be checked over time. In the present computations the conservation of mass is met with a maximum relative error of $10^{-4.5}$ and the conservation of energy is met with a maximum relative error of $10^{-3}$. The present model is robust enough to avoid the use of any smoothing or regridding. Once the change of variables is performed, the set of source singularities and their mirror images with respect to the horizontal axis in the transformed $w$-plane makes it possible to easily account for the impermeability condition on the walls.

In the sequel, we shall use complex functions to describe the potential flow. The total complex potential is denoted $F$ which is a function of the complex coordinate $z$. The complex potential is expanded as a summation of sources distributed in the vicinity of the boundaries and located outside the fluid domain. Its real part yields the velocity potenlial $\Phi$

$$
F(z, t)=\sum_{j=1}^{N} q_{j}(t) G\left(w, W_{j}(t)\right)+\left(z-z_{A}\right) \chi_{A}(t)
$$


where $\chi_{A}(t)$ is the conjugate of the complex translational velocity of point $A . W_{j}(t)$ is the complex coordinate of the $j^{t h}$ source point attached to the free surface in the transformed plane. $Z_{j}(t)$ is the complex coordinate of the $j^{\text {th }}$ source points in the physical plane located outside the fluid domain and surrounding all the fluid boundaries. $W_{j}(t)$ are also the image of the $j^{\text {th }}$ source $Z_{j}(t)$ in the physical plane by using the mapping function is $W_{j}=g\left(Z_{j}\right)$ defined by $(3)$. The intensities $q_{j}(t)$ are obtained from the velocity potential updated at the free surface by solving a time differential system for $\varphi$. $G$ is the complex potential of a source (and its mirror image) calculated in the transformed plane $w$ as follows

$$
G\left(w, W_{j}\right)=G_{j}=\log \left(w-W_{j}\right)+\log \left(w-\overline{W_{j}}\right)
$$

where the second contribution allows to account for the impermeability condition along the surface of the tank. The other two contributions in $F(z, t)$ are the equivalent complex expressions of the Stokes-Joukowski velocity potential $\phi$ defined in (2).

For two-dimensional configurations, the pressure $p(x, y, t)$ meets the following two equations at the free surface,

$$
p=0, \quad \frac{d p}{d t}=0
$$

It is also useful to formulate the Euler equation for the velocity $\vec{u}=(u, v)$

$$
\vec{u}_{, t}+(\vec{u} \cdot \vec{\nabla}) \vec{u}+\frac{\vec{\nabla} p}{\rho}=\vec{g}
$$

in a equivalent complex form as follows

$$
F_{, z t}+F_{, z^{2}} \overline{F_{, z}}+\frac{p_{, z}}{\rho}-i g=0
$$

where $p_{, z}$ is the conjugate of the pressure gradient $p_{, z}=p_{, x}-i p_{, y}$ The points where the pressure is stationary follow from the conditions $p_{, x}=p_{, y}=0$. The location of vanishing pressure gradient is also that of a saddle point or local maximum of pressure. The distinction between these two cases is done by evaluating the Gaussian curvature and the mean curvature. The latter is simply the Laplacian of the pressure and it reads

$$
\Delta p=p_{, x^{2}}+p_{, y^{2}}=4 p_{, z \bar{z}}=-2 \rho F_{, z^{2}} \overline{F_{, z^{2}}}=-2 \rho\left|F_{, z^{2}}\right|^{2}
$$

where $F_{, z^{2}}$ follows from

$$
F_{, z^{2}}=u_{, x}-i u_{, y}
$$

having in mind the identities that follow from incompressibility $\left(u_{, x}+v_{, y}=0\right)$ and irrotationality $\left(u_{, y}-v_{, x}=0\right)$. It is worth reminding that $F_{, z \bar{z}}=0$ since $F$ is analytic, but it is not the case for the pressure whose Laplacian is not zero in equation (9). The Gaussian curvature is given by the formula

$$
\Omega=\frac{1}{\rho^{2}}\left(p_{, x^{2}} p_{, y^{2}}-p_{, x y}^{2}\right)=\left|F_{, z^{2}}\right|^{4}-\left|F_{, z^{3}} \bar{F}_{, z}+F_{, z^{2} t}\right|^{2}
$$

The change of sign of $\Omega$ indicates a change of the property of the pressure at a point where its gradient drops to zero. When $\Omega<0$ a zero of the pressure gradient indicates a saddle point. When $\Omega>0$ a zero of the pressure gradient indicates a local maximum. When this occurs in a close vicinity to a free surface, great pressure gradients are expected and then appearance of a cusp as well due to great acceleration. The appearance of a cusp along the free surface can be identified by calculating the radius of curvature. To evolve continuously 
along the free surface, the arclength $\sigma$ is calculated from the left wall to the right wall. A cartesian coordinate $(x, y)$ of the fluid particle at the free surface only depend on this arc length and time $t$. The radius of curvature reads

$$
R_{c}=\frac{\left(\left(x_{, \sigma}\right)^{2}+\left(y_{, \sigma}\right)^{2}\right)^{3 / 2}}{x_{, \sigma} y_{, \sigma^{2}}-y_{, \sigma} x_{, \sigma^{2}}}
$$

The change of sign of $R_{c}$ is of interest. It means that there is an inflexion point. In practice, only the change of sign of the denominator $x_{, \sigma} y_{, \sigma^{2}}-y_{, \sigma} x_{, \sigma^{2}}$ is examined.

\section{Discussion}

The objectives of the present numerical simulations, are to arrive at a plunging breaker close to the left vertical wall of the rectangular tank. For that a parametric study has been achieved (see Scolan, 2018) where a large range of plunging breakers associated with a critical jet are simulated. On the basis of this parametric study, we analyze here one of the critical jet simulations. The applications of the present study participates to the prediction of the fluid critical kinematics in a liquefied natural gas carrier (see Brosset et al, 2009; Dias and Ghidaglia, 2018).

The length of the tank is $L=1.08 \mathrm{~m}$ and it is filled up to $h=0.22833 \mathrm{~m}$. The fluid critical kinematics is obtained in the tank by forcing its motion. Here the motion consists in an horizontal cycle starting from rest (see Karimi et al, 2016). Figure (2) shows the time variations of the displacement, velocity and acceleration. By stopping the forced motion at some intermediate instants, a parametric study makes it possible to capture a large variety of critical jets along the surface of the overturning crest in the barrel. The retained case is a restart at instant $t_{o}=1.63 \mathrm{~s}$ which is indicated with a vertical line in figure (2). It means that after this instant, no more energy is injected in the fluid. As the motion of the tank is not forced any longer, the complex potential $F(z, t)$ that describes the flow reduces to the first contribution in equation (4).

We consider a restart with a given quantity of kinetic energy and potential energy in the fluid. This configuration is unfortunately difficult to reproduce experimentally due to the necessity to stop the tank motion instantly. For these precise initial conditions (deformation of the free surface and distribution of velocity potential on this free surface), the parametric study shows that we end up with a relatively large size of critical jet as illustrated in figure (3).

For the discussion, we choose two instants $t_{1}=0.1506 \mathrm{~s}$ and $t_{2}=0.1542 \mathrm{~s}$, at which we analyze the properties of the fluid kinematics and dynamics. The corresponding free surface profiles are plotted in figure (3). The first instant $t_{1}=0.1506 \mathrm{~s}$ precedes the start of the growing jet. It is easy to identify this instant by analyzing the change of sign of the radius of curvature along the free surface. The second instant $t_{2}=0.1542 \mathrm{~s}$ corresponds to a well developed jet.

Figure (4) shows snapshots of the three vector fields that appear in Euler equation (7), namely the acceleration $\vec{u}_{, t}$, the convective component $(\vec{u} \cdot \vec{\nabla}) \vec{u}$ and pressure gradient $\frac{\vec{\nabla} p}{\rho}$ at instant $t_{1}$. Closer views focus on the region where maximum is reached. The sum of these three quantities is exactly equal to the vector gravity $\vec{g}$ oriented vertically downwards. It is worth reminding that the pressure gradient at the free surface is parallel to the normal and oriented inside the fluid domain consistently with the fact the pressure is nil at the free surface and positive inside the fluid domain. It should be noted that the normal gradient of the pressure is great in the barrel up to the top of the barrel where the tangent is horizontal. Up to this instant for which the free surface is very smooth in the barrel, these features 
are quite consistent with the observations made in Peregrine et al (1980), Yasuda (1997) or Scolan and Guilcher (2019). In figure (4) we emphasize the points of the maximum positive Gaussian curvature $\Omega$ and the minimum of the pressure gradient $\|\vec{\nabla} p\|$. The former point is identified from the isocontour of positive $\Omega$ in figure $(5 \mathrm{f})$. The latter point can be picked up from the isocontour of $\|\vec{\nabla} p\|$ plotted in figures (5c) and its closer view (5d). This is also the maximum of the pressure field plotted in figures (5a) and its closer view (5b). In figure (4) we add a closed curve that delimits the inner region where the Gaussian curvature is positive. That confirms that the point where $\|\vec{\nabla} p\|$ vanishes is clearly a maximum and not a saddle point. The point of maximum Gaussian curvature is very close to the free surface. The spatial distributions of $\|\vec{\nabla} p\|$ and $|\Omega|$ are quite identical on the two sides of the point where the acceleration reaches its maximum. Figure $(5 \mathrm{~g})$ shows the isocontour of the acceleration modulus. As the local variation is very great, the logscale of acceleration is used to distinguish the isocontours. The mean curvature (plotted in figure 5h) reaches its (negative) maximum along the free surface at the same point of maximum acceleration. The spatial distributions of ||$\vec{u}_{, t}||$ and $|\Delta p|$ are quite identical around the point where the acceleration reaches its maximum. Here again logscale of the mean curvature is plotted to capture the great variation of this quantity around its maximum.

In order to illustrate the relative importance and the local orientation of the vector components in Euler equation (7), their cartesian components are drawn in figure (6). The origin of the coordinate system corresponds to the point where the maximum positive Gaussian curvature is reached at instant $t_{1}=0.1506 \mathrm{~s}$. The vectorial sum of the three vectors $\left(\vec{u}_{, t}\right.$, $\left.(\vec{u} \cdot \vec{\nabla}) \vec{u}, \frac{\vec{\nabla} p}{\rho}\right)$ is equal to the gravity $\vec{g}$. It is clear that gravity is negligible there since the length of vector $\vec{g}$ is roughly the size of the plot divided by 100. The Eulerian acceleration $\vec{u}_{, t}$ and convective term $(\vec{u} \cdot \vec{\nabla}) \vec{u}$ are in opposite directions. Their sum yields the Lagrangian acceleration. This quantity is very great in the close vicinity of the maximum of Gaussian curvature and its main contribution originates from the convective term. This fact might be counter intuitive since in Bernoulli equation, written at the free surface where the pressure vanishes, the contribution of $\left|\phi_{, t}\right|$ is always greater than the contribution of $\frac{1}{2}(\vec{\nabla} \phi)^{2}$.

Figures (7) and (8) show the same quantities than in figures (4) and (5) respectively but at a later instant when the critical jet has already well increased in size. The features of the kinematics and dynamics of the flow remain quite similar but the order of magnitude increases significantly, with a factor 10 approximately.

The time variations of $\|\vec{\nabla} p\|_{\min }, p_{\max }$ and $\Omega_{\max }$ are plotted in figure (9). We start the analysis at instant $t=0.1342 \mathrm{~s}$ when the dynamics increases rapidely. At this instant $\|\vec{\nabla} p\|_{\text {min }}$ is of the order of gravity $g=9.81 \mathrm{~m} / \mathrm{s}^{2}$. Then this quantity regularly decreases and vanishes at the instant when the critical jet appears, roughly $t \approx 0.1502 \mathrm{~s}$. It should be noted that the critical jet appears precisely when the quantity $p_{\max }$ starts to increase significantly. The maximum of $p_{\max }$ is reached at instant $t=0.15194 \mathrm{~s}$ and it is of the order of the hydrostatic pressure $\rho g h$. This pressure is rather small compared to maximum pressure computed when a flip-through (for example) is observed. That possibly means that the presence of a wall (material surface or plane of symmetry) may play an important role in the increase of the pressure. The maximum of $\Omega_{\max }$ is approximately reached at instant $t=0.1512 \mathrm{~s}$ and it is of the order of $10^{12} \mathrm{~s}^{-2}$.

It appears that the combination of a maximum pressure and a positive Gaussian curvature in a close vicinity of the free surface is the necessary condition for the appearance of a local and concentrated great fluid kinematics. As a consequence a sharp corner appears and grows continuously. It is therefore important to track over time these two properties of the pressure field before the sharp corner appears. Figure (10a) shows the time tracking of 
the locations of the maximum pressure $p_{\max }$ (when it exists effectively) and the minimum modulus of the pressure gradient $\|\vec{\nabla} p\|_{\text {min }}$. The two trajectories are superposed after the instant $t=0.1502 \mathrm{~s}$. These points are otherwise embedded in a region where the Gaussian curvature is positive. That means that we have a local maximum pressure indeed. Beyond the instant $t=0.1502 \mathrm{~s}$ and until the end of the numerical simulation, these points are superposed, indicating the persistence of the local maximum. Before the instant $t=0.1502 \mathrm{~s}$, the location $\|\vec{\nabla} p\|_{\text {min }}$ follows a straight path. It should be noted that the corresponding trajectory is continuous in space.

A closer view of the trajectories is done in figure (10b). In addition, the locations of the maximum positive Gaussian curvature $\Omega_{\max }$ are plotted. It is observed that the Gaussian curvature has positive values close to the free surface, well before the appearance of the other maxima, but without provoking a cusp at the free surface. It can be observed that the region of positive Gaussian curvature intersects the free surface precisely where the free surface profile has local change of curvature. This point is better described later in this discussion.

The location of $\Omega_{\max }$ suddenly migrates to the region where the pressure gets its own maximum. The first local maximum of pressure occurs clearly inside the fluid (relatively) far from the free surface. Finally, when the critical jet appears approximately at instant $t_{1}=0.1506 s$, the trajectories of both maxima stay within a close vicinity. It should be noted that $\Omega_{\max }$ occurs at the root of the critical jet as if this great local variation of the pressure up to its local maximum maintains the development of the critical jet.

The local variation of the pressure between the free surface and the point where $\Omega_{\max }$ is reached, is hence very rapid. As a matter of fact, the pressure is nil at the free surface, the normal gradient of the pressure is finite and great and the curvature varies spatially very rapidely and changes sign as well. In order to illustrate the sharp front of the pressure in the close vicinity of the free surface, we fit a function between the two points corresponding to $\Omega_{\max }$ and $p_{\max }$ at instant $t=0.1512 \mathrm{~s}$. The slope at the free surface is very steep, while a threshold is reached rapidely, hence suggesting a hyperbolic tangent function. Figure (11) compares the function $p(\sigma) / \rho=\alpha \tanh (\beta \sigma)$ with the numerical pressure distribution between the two points located at $\Omega_{\max }$ and $p_{\max } / \rho, \sigma$ being the distance from the free surface. It is obtained for $\alpha=2.05 \mathrm{~m}^{2} / \mathrm{s}^{2}$ and $\beta=484 \mathrm{~m}^{-1}$.

In order to get an overview of the acceleration at the free surface, figure (12) shows the temporal-spatial variations of the Langrangian acceleration $\left\|\frac{d \vec{u}}{d t}\right\|$ at the free surface for the sequence of the simulation as it varies significantly. This modulus is exactly the normal gradient of the pressure $\left|p_{, n} / \rho\right|$ provided the acceleration gravity is negligible. This is indeed the case over a large arclength of the free surface and for the last stages of the simulation after $t \approx 0.15 \mathrm{~s}$. At a given instant the acceleration reaches its maxima at two points on both sides of the tip of the critical jet. The black marks track the main maximum which is located somewhere in the secondary barrel (between the critical jet and the main crest). The second maximum (less sharp) occurs at the top of the main barrel. Between the two maxima of acceleration there is the tip of the critical jet where the velocity reaches its maximum. It should be noted that the time variation of the acceleration shows that its maximum (about $2700 \mathrm{~m} / \mathrm{s}^{2}$ ) is reached before the end of the simulation at instant $t=0.153 \mathrm{~s}$ consistently with the time variation of the maximum of pressure shown in figure (9).

The radius of curvature along the free surface profile is also a variable that gives some insights into the posed problem. This radius follows from the definition (12). Figure (13a) shows the last free surface profiles of the simulation and some of them are emphasized. They clearly show a change of sign of the curvature sufficiently before the appearance of the critical jet. The corresponding radius of curvature is plotted in figure (13b), actually its absolute 
value. The infinite radius means that the denominator of $R_{c}$ in equation (12) vanishes. The plot covers the last stages of the simulation up to the full development of the critical jet that appears at $t=0.1506 \mathrm{~s}$. Before that instant the free surface profile shows a change of curvature starting at $t=0.138 \mathrm{~s}$ and ending just before the appearance of the critical jet.

The location in time and space of the zeros of $\Omega$ and $R_{c}$ is represented in figure (14). It is a kind of upper view of figure (13b). There is a strong correlation between the zeros of $\Omega$ and $R_{c}$. It is reminded that the zeros of $\Omega$ bounds the region where the Gaussian curvature is positive. The figure hence shows that even a slight growth of the free surface is associated with a positive Gaussian curvature, that is to say a potentially significant cusp if the pressure gradient should vanish there. Geometrically this can be explained if we consider the pressure surface as a steep "cliff" close to the free surface, say almost vertical and flat. If we pull somewhere the line that describes the foot of the cliff (thus imitating a local growth), that influences the surface of the cliff above and its Gaussian curvature. The formal proof is still to be done.

\section{Conclusion}

Similar free surface flow computations have also been done but for less strong forcing. This is possible by tuning the duration of the forcing, thus less energy is injected in the fluid but still an overturning crest is yielded. Nevetheless the critical jet does not appear. That is because the region of positive Gaussian curvature and the region of vanishing pressure gradient in the vicinity of the free surface do not overlap.

It is observed that when the pressure gradient vanishes close to the free surface and when this precisely occurs in a region where the Gaussian curvature reaches high positive values as close as possible to the free surface, then a cusp is likely to occur which is accompanied with a great kinematics. This observation confirms what Longuet-Higgins (1980a) anticipated theoretically.

In future works we shall analyze other configurations where overturning crest does not necessarily precede the appearance of a critical jet. An example is described in LonguetHiggins (2001a), and the critical jet is known as the "bazooka" effect. Using again a horizontal cyclic motion of a rectangular tank, Scolan and Etienne (2018) describe such a phenomenon which is followed by a flip-through event (see Cooker and Peregrine, 1990). The resulting kinematics are very large. A preliminary analysis confirms that the main characteristics described in this note are also met for these violent free surface flows with critical jets. 


\section{References}

Longuet-Higgins, M.S. 1980, A Technique for Time-Dependent Free-Surface Flows. Proceedings of the Royal Society of London. Series A, Mathematical and Physical Sciences, 371, 441-451.

Longuet-Higgins, M.S. 1980, On the forming of sharp corners at a free surface. Proceedings of the Royal Society of London. Series A, Mathematical and Physical Sciences, 371, 453-478.

Longuet-Higgins, M.S. 1993, Highly accelerated, free-surface flows. J. Fluid Mech., 248, 449-475.

Longuet-Higgins, M.S. And Dommermuth, D.G. 2001, Vertical jets from standing waves. II. Proceedings of the Royal Society of London. Series A, Mathematical and Physical Sciences, 457, 2137-2149.

Zeff, B. W., Kleber, B., Fineberg, J. \& Lathrop, D. P., 2000, Singularity dynamics in curvature collapse and jet eruption on a fluid surface. Nature, 403 401-404.

Cooker, M.J., Peregrine, D.H., 1990, A model of breaking wave impact pressures. Proc. 22nd Conf. Coastal Engineering, Holland ASCE, 1473-1486.

Cooker, M.J., 2001, Unsteady pressure fields which precede the launch of free-surface liquid jets. Proc. R. Soc. Lond. A., 458, 473-488.

Cooker, M.J., 2010, The flip-through of a plane inviscid jet with a free surface. J. Eng. Math, 67, 137-152.

Longuet-Higgins M. S. , 2001, Vertical jets from standing waves; the bazooka effect, IUTAM Symposium on Free Surface Flows, A.C. King and Y.D. Shikhmurzaev (eds.), Kluwer Academic Publishers, 195-203.

Grilli, S.T., Subramanya, R., 1996, Numerical modeling of wave breaking induced by fixed or moving boundaries. Computational Mechanics, 17, 374-391.

Scolan Y.-M., Hay A. \& Brosset L., 2016 Some aspects of high kinematics in breaking waves due to sloshing. $31^{\text {st }}$ International Workshop on Water Waves and Floating Bodies, Plymouth, USA, April 2016,, 157-160.

Scolan Y.-M., 2018, Critical Free Surface Flows in a Sloshing Tank, Journal of Advanced Research in Ocean Engineering, 4(4), 163-173 http://dx.doi.org/10.5574/JAROE.2018.4.4.163

Scolan, Y.-M., 2010, Some aspects of the flip-through phenomenon: A numerical study based on the desingularized technique J. Fluid Struc., 26, Issue 6, 918-953.

Tuck E.O., 1998, Solution of Nonlinear Free-Surface Problems by Boundary and Desingularised Integral Equation Techniques, Invited Lecture, Computational Techniques and Applications: CTAC'gry Eds J. Noye, M. Teubner, A. Gill, World Scientific, Singapore, $11-26$.

Faltinsen O.M., Rognebakke, O., Lukowsky I, and Timokha A., 2000, Multidimensional modal analysis of nonlinear sloshing in a rectangular tank with finite water depth, J. Fluid Mech., 407, 201-234 
Scolan Y.-M. \& L. Brosset, 2017, Numerical Simulation of Highly Nonlinear Sloshing in a Tank Due to Forced Motion, International Journal of Offshore and Polar Engineering, 27, Issue 1, 11-17.

Spiegel, M.R., 1999, Complex variable with an introduction to conformal mapping and its applications. Schaum's outlines series, McGraw-Hill, 313 pages, $32^{\text {nd }}$ edition.

Cao, Y., Schultz, W.W., Beck, R.F., 1991, A three-dimensional desingularized boundary integral method for potential problems, International Journal Numerical Method in Fluids, 11, 785-803.

Brosset, L., Mravak, Z., Kaminski, M. Collins, S. and Finnigan, T., 2009, Overview of SLOSHEL project, Proc. ISOPE, Osaka, Japan

Dias F. And Ghidaglia J.-M., 2018, Slamming: Recent Progress in the Evaluation of Impact Pressures Annual Review of Fluid Mechanics, 50, 243-273.

Karimi M.R., L. Brosset, J.-M. Ghidaglia, M.L. Kaminski, 2016, Effect of ullage gas on sloshing, Part II: Local effects of gas-liquid density ratio. European Journal of Mechanics B/Fluids, 57, 82-100.

Peregrine, D., Cokelet, E., \& McIver, P. 1980, The fluid mechanics of waves approaching breaking. Coastal Engineering Proceedings, 1(17), 31.

Yasuda T., Mutsuda H., Mizutani N. 1997, Kinematics of overturning solitary waves and their relations to breaker types Coastal Engineering, 29, Issues 3-4, 317-346.

Scolan Y.-M. And Guilcher P.M., 2019, Wave Kinematics in a Two-Dimensional Plunging Breaker, Water Waves, https://doi.org/10.1007/s42286-019-00013-w

Scolan Y.-M. And S. Etienne, 2018, Critical jets in a breaking wave (in french), Proceedings of the $16^{\text {th }}$ Journées de l'Hydrodynamique, Marseille, France. 


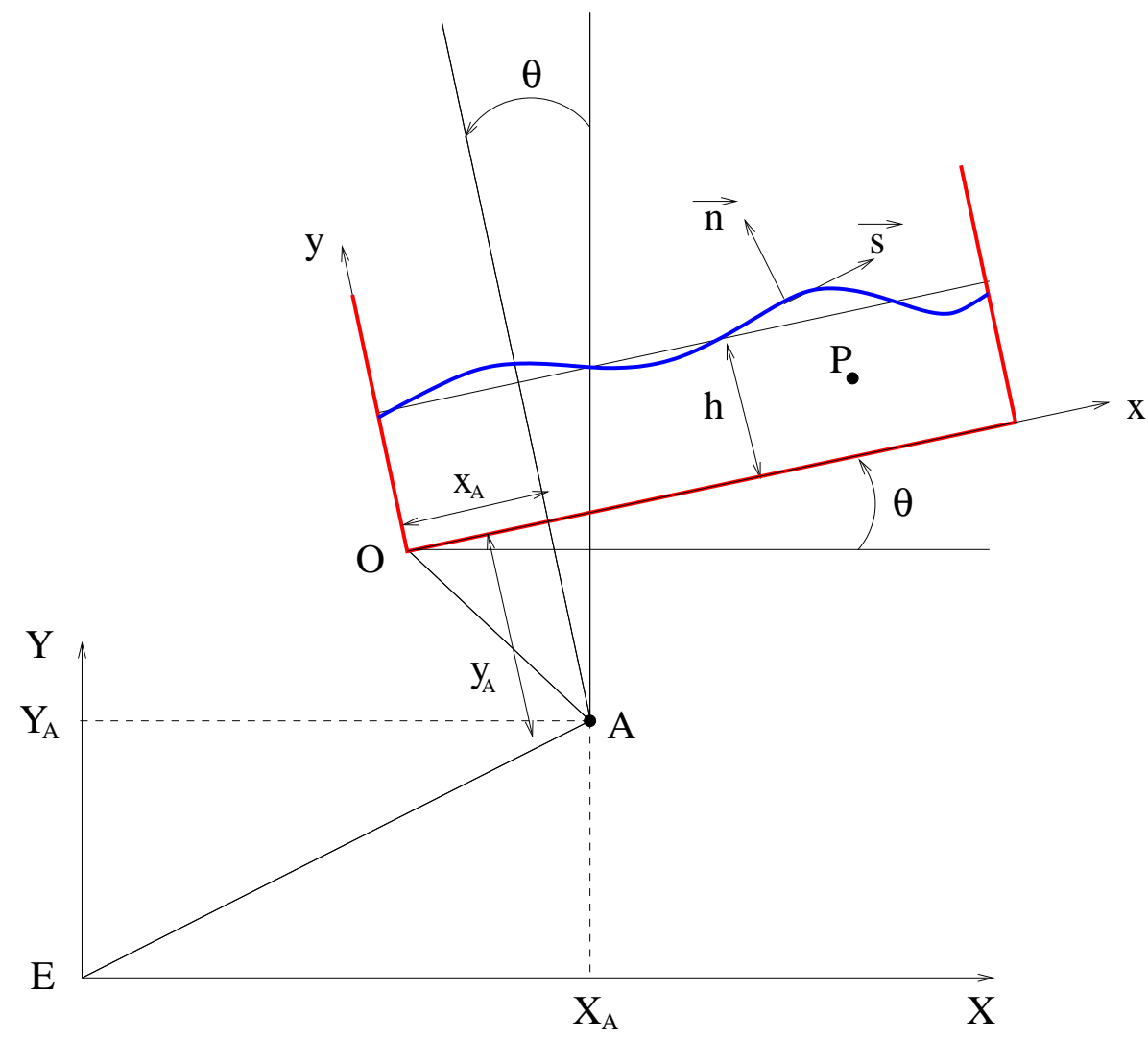

Figure 1: Notations used to describe the motion of the moving tank. $\left(X_{A}, Y_{A}\right)$ are the cartesian coordinates of the point of rotation $A$ in the earth fixed coordinate system $(E, X, Y) .\left(x_{A}, y_{A}\right)$ are the cartesian coordinate of the point of rotation $A$ in the coordinate system $(O, x, y)$ attached to the moving tank. The angle of rotation is $\theta$. 


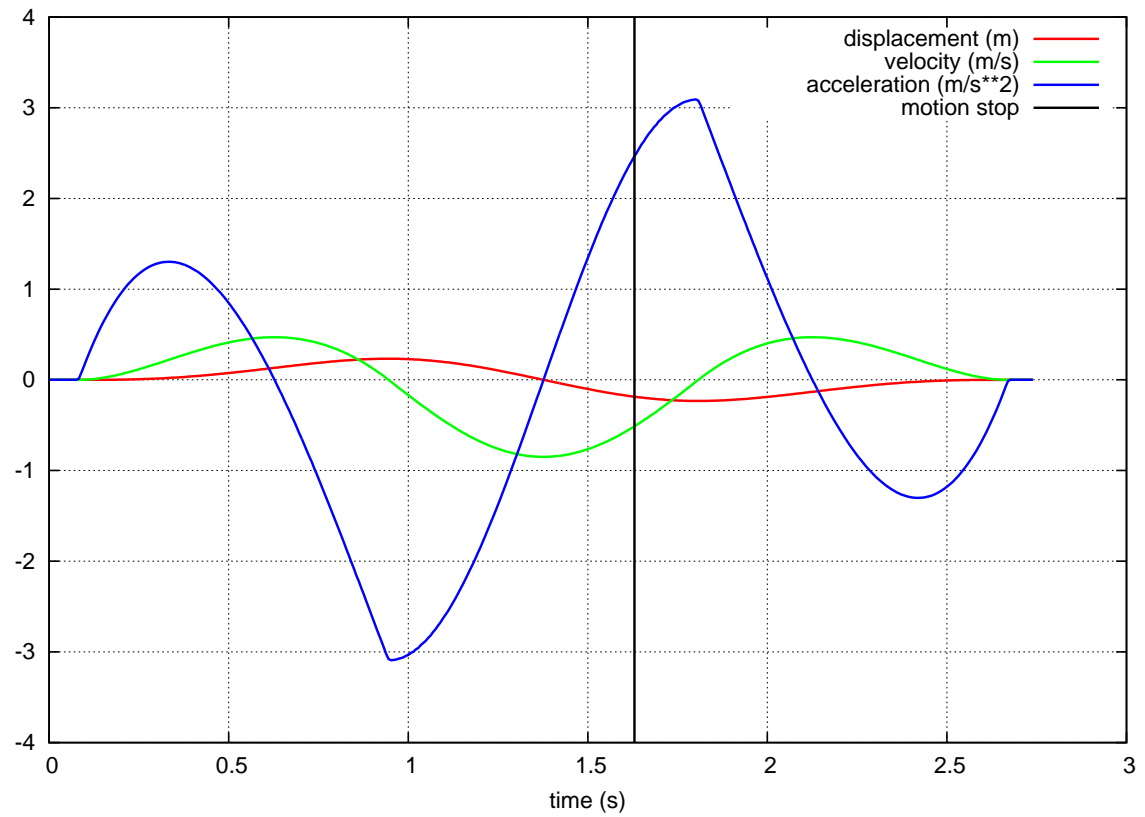

Figure 2: Horizontal forcing motion of the rectangular tank. Displacement, velocity and acceleration. The vertical line shows indicates the instant $\left(t_{o}=1.63 \mathrm{~s}\right)$ at which the motion is stopped. 


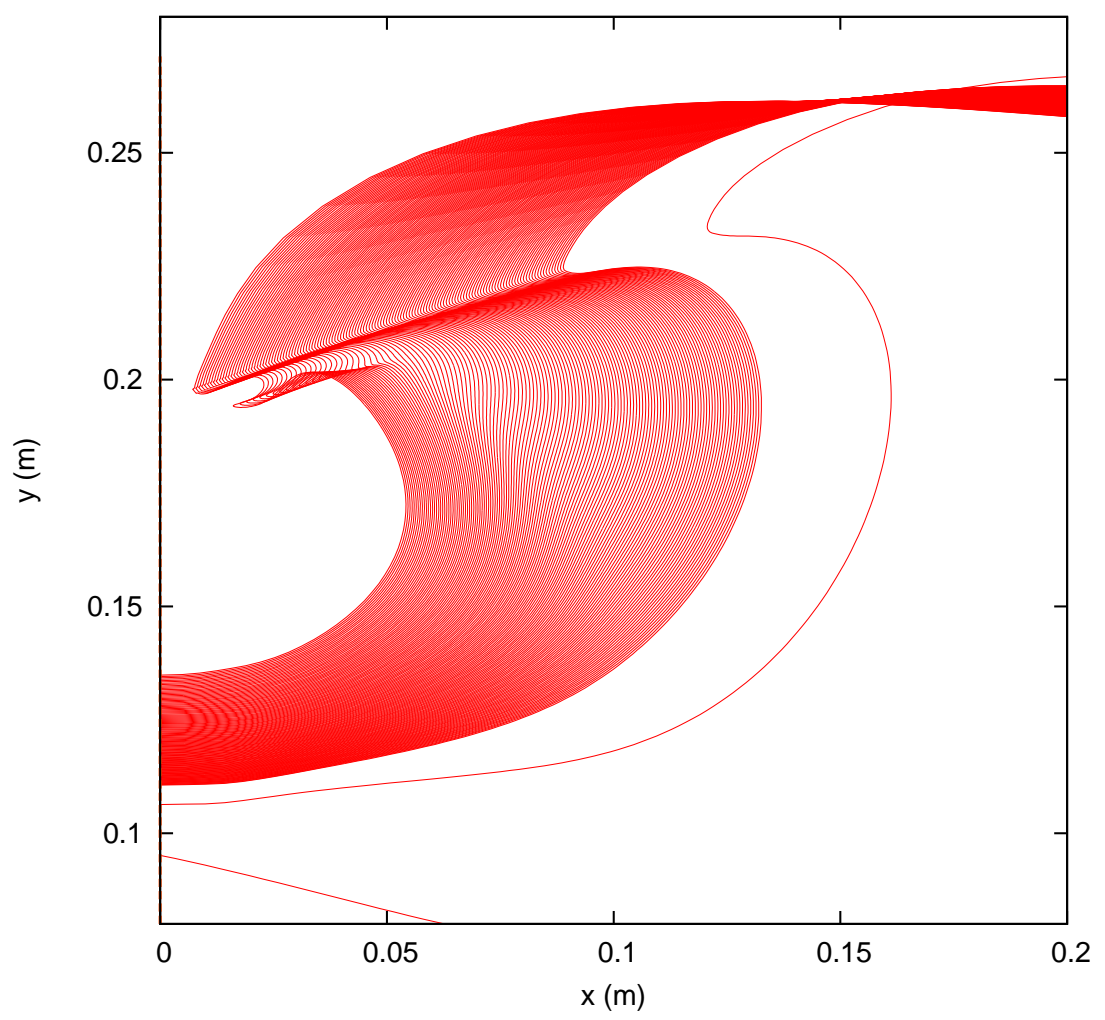

(a)

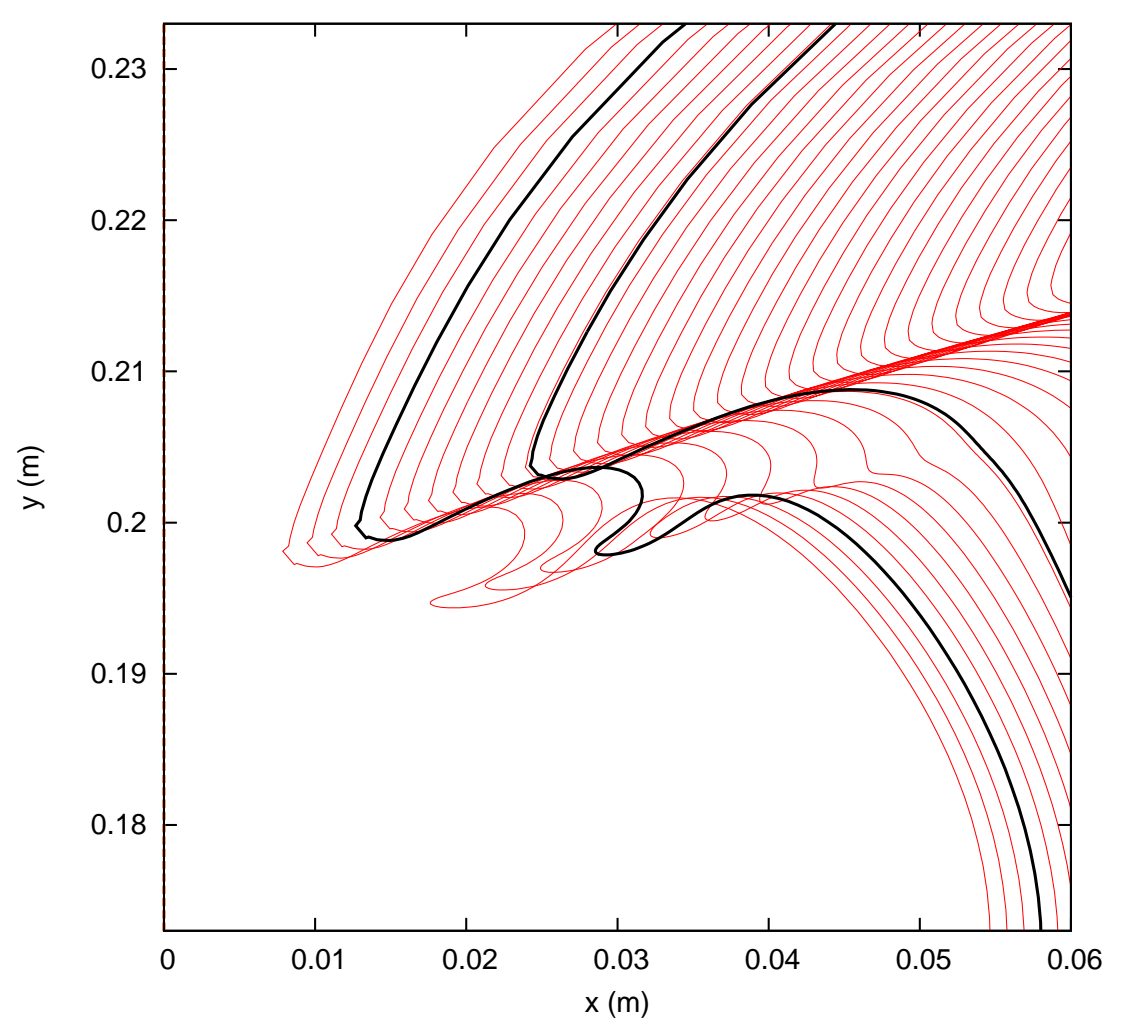

(b)

Figure 3: Successive free surface profiles simulated from a restart at instant $t_{o}=1.63 \mathrm{~s}$ with a horizontal forced motion of the tank plotted in figure (2). The time step between each profile is (a) $210^{-4} s$ and (b) $510^{-4} \mathrm{~s}$. The profiles at the two instants $t_{1}=0.1506 \mathrm{~s}$ and $t_{2}=0.1542 \mathrm{~s}$ are superimposed. 
(a)
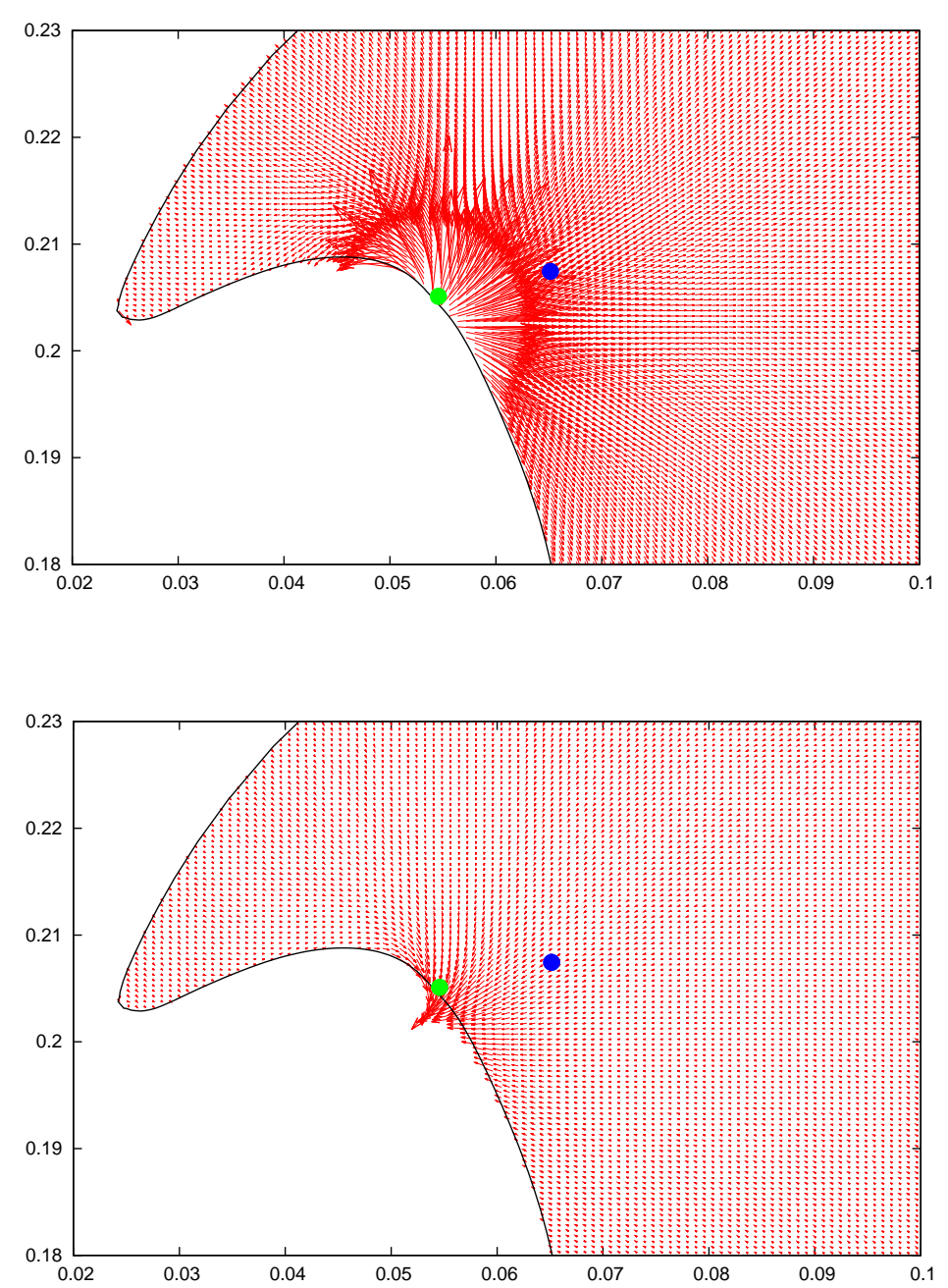

(b)

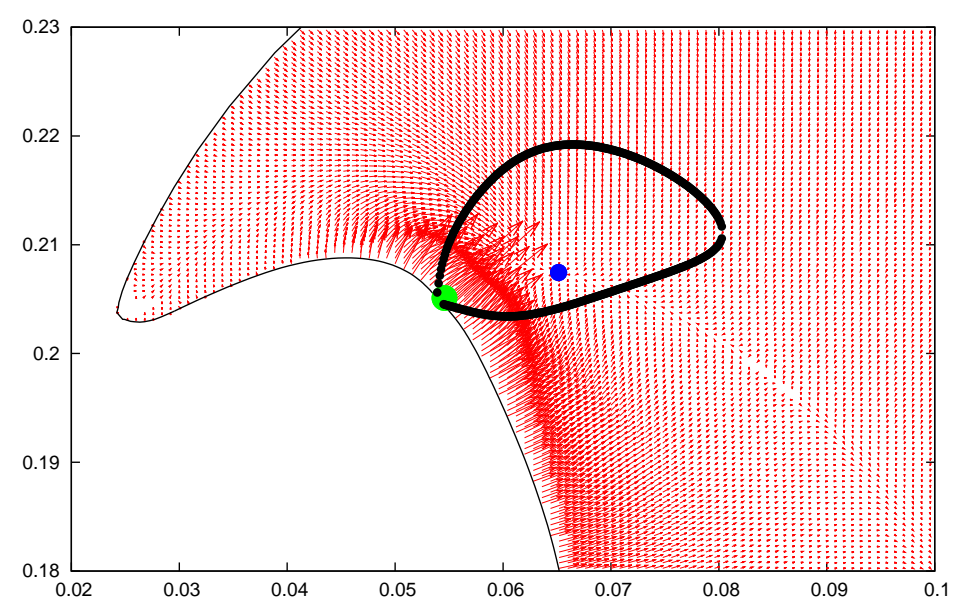

(c)
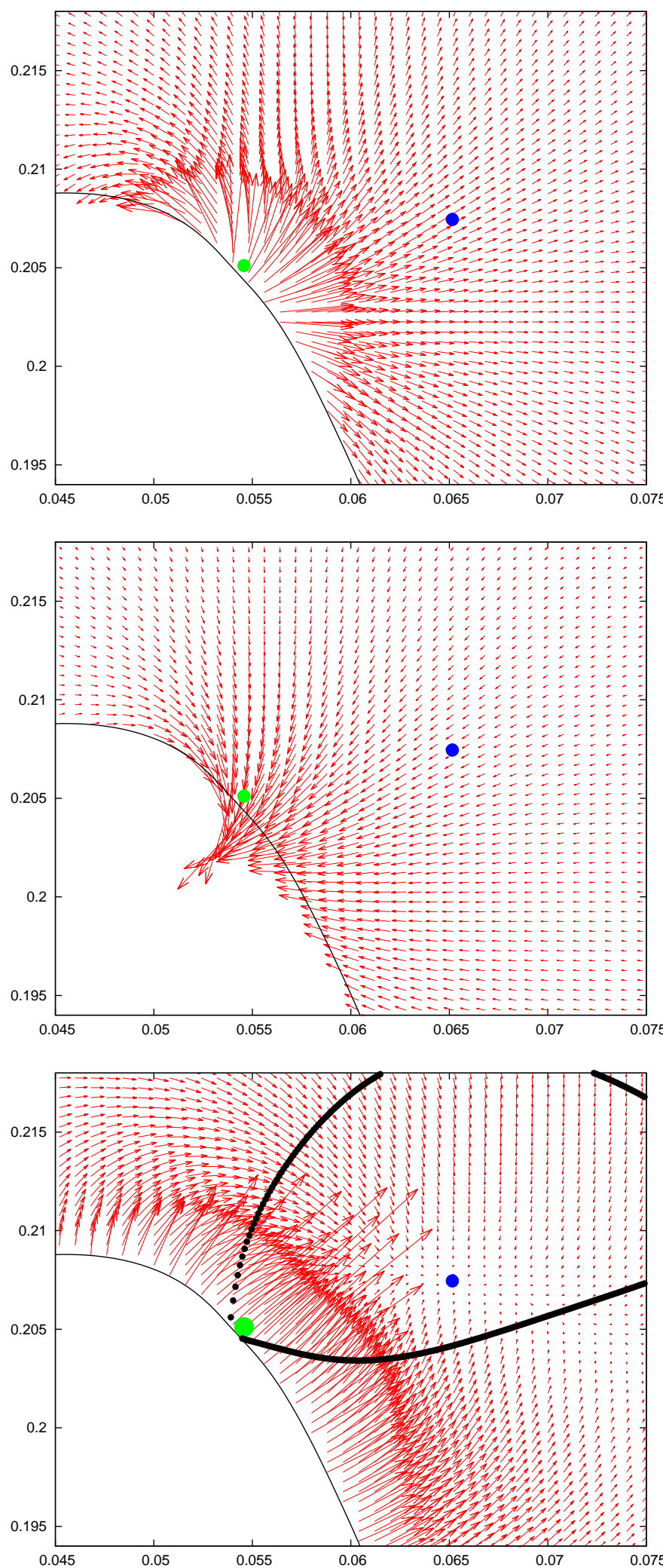

Figure 4: Vector fields of the (a) acceleration $\vec{u}_{, t},(\mathrm{~b})$ convective term $(\vec{u} \cdot \vec{\nabla}) \vec{u}$ and (c) pressure gradient $\frac{\vec{\nabla} p}{\rho}$, at instant $t_{1}=0.1506 \mathrm{~s}$. The right figures are closer views of the left figures. The two cartesian components of the vector are divided respectively by $210^{5}, 10^{6}$ and $10^{5}$ for (a), (b) and (c). The units of the vectors are $m^{2} / s^{2}$, Units of the horizontal and vertical coordinates: $m$. The green and blue marks are the locations of the maximum positive value of the Gaussian curvature $\Omega$ and the minimum of $\|\vec{\nabla} p\|$ respectively. The closed curves delimit the inner region where the Gaussian curvature is positive. 


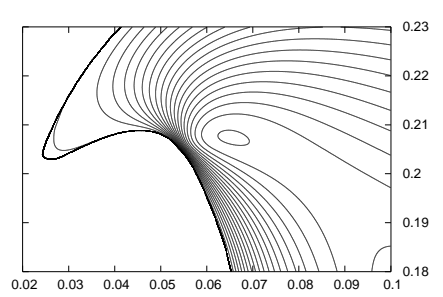

(a)

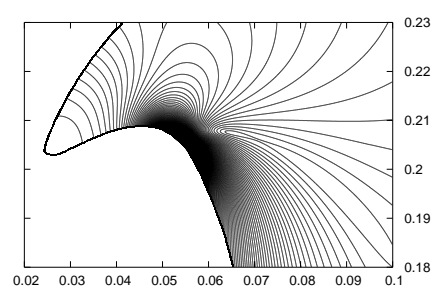

(c)

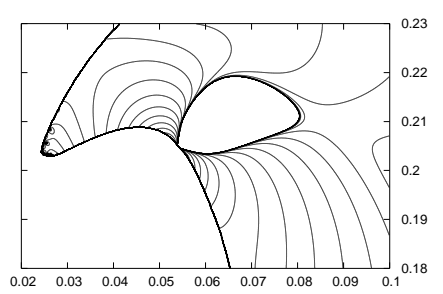

(e)

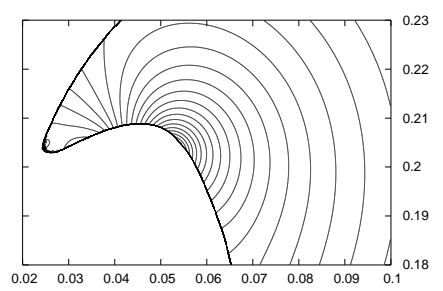

(g)

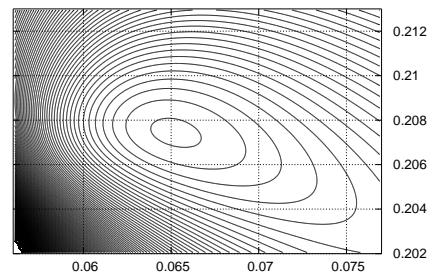

(b)

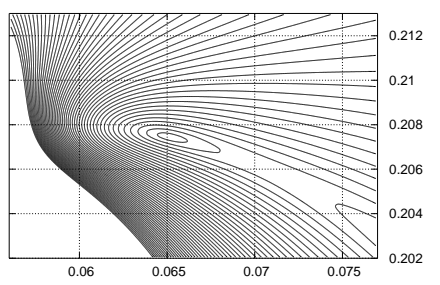

(d)

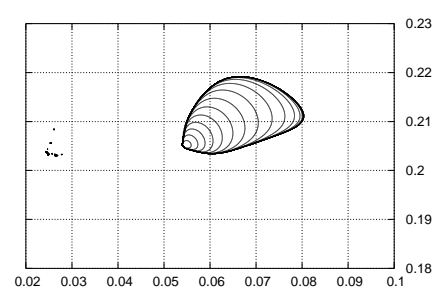

(f)

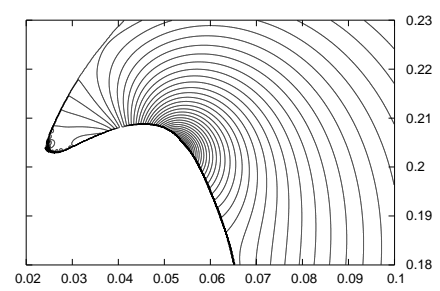

(h)

Figure 5: Isocontours of scalar fields at instant $t_{1}=0.1506 \mathrm{~s}$. The three digits in brackets give the minimum, the step and the maximum of each isocontour plot. (a), (b) : pressure $(0,0.1,1.912),(0,0.01,1.912)(\mathrm{c}),(\mathrm{d})$ : modulus of the pressure gradient, $(0,5,1000),(0,2,100)(\mathrm{e})$, (f) : respectively negative and positive Gaussian curvature $(\log 10),(-20,0.5,0),(0,0.5,15)(\mathrm{g}): \log$ of the modulus of the acceleration, $(0,0.1,4),(\mathrm{h}): \log$ of the mean curvature. $(0,0.1,9)$, Units of the horizontal and vertical coordinates: $m$. 


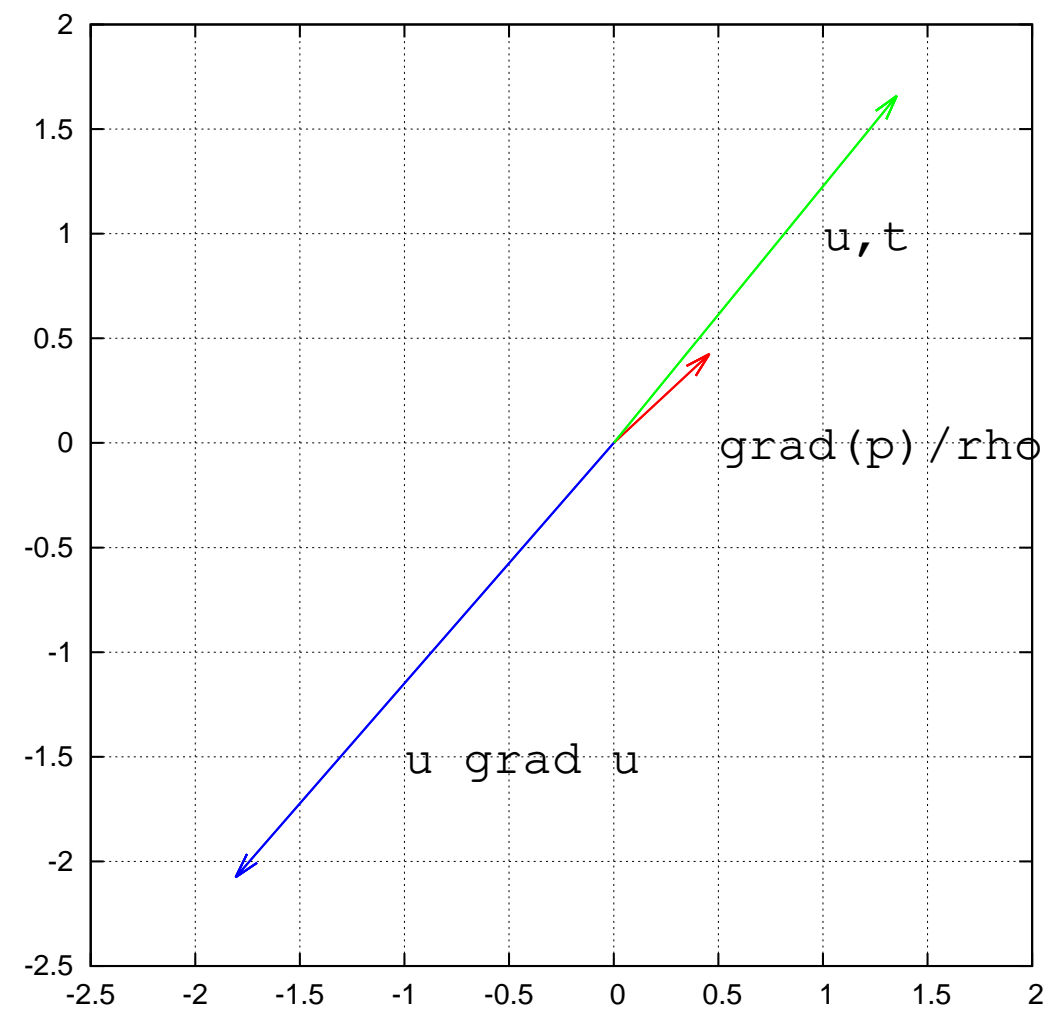

Figure 6: Vector components of the acceleration $\vec{u}_{, t}$, convective term $(\vec{u} \cdot \vec{\nabla}) \vec{u}$ and pressure gradient $\frac{\vec{\nabla} p}{\rho}$, centered at the point where the maximum positive Gaussian curvature is reached at instant $t_{1}=0.1506 s$. The scale is $1: 1000$ and unit is $m / s^{2}$. 


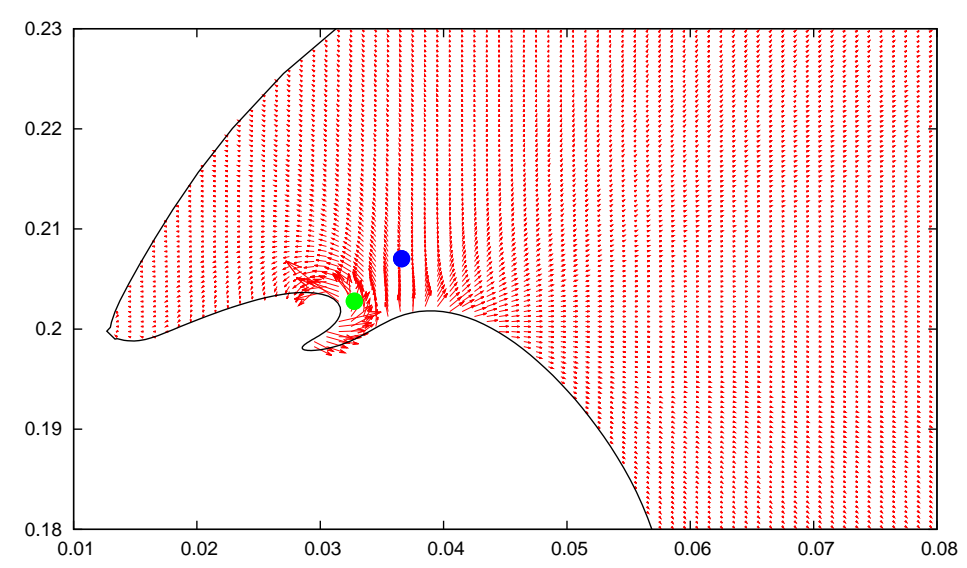

(a)

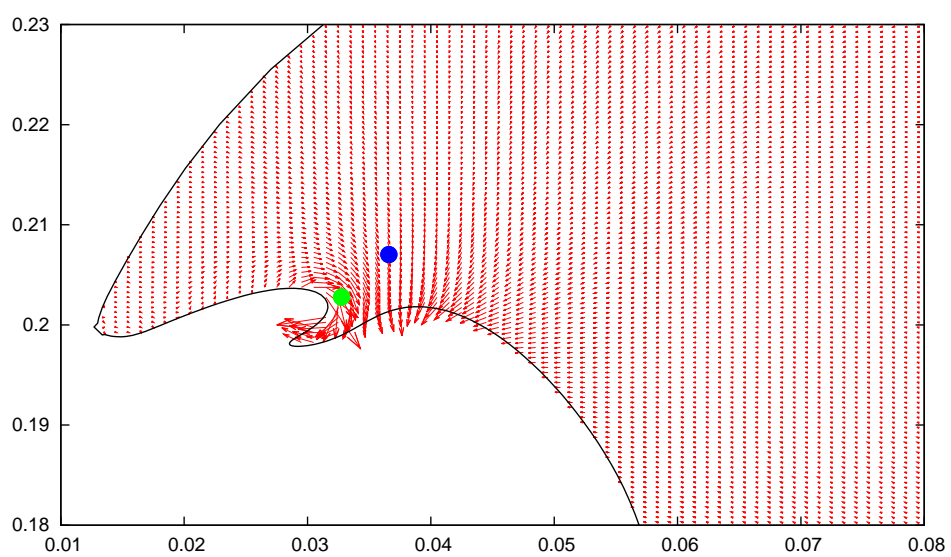

(b)

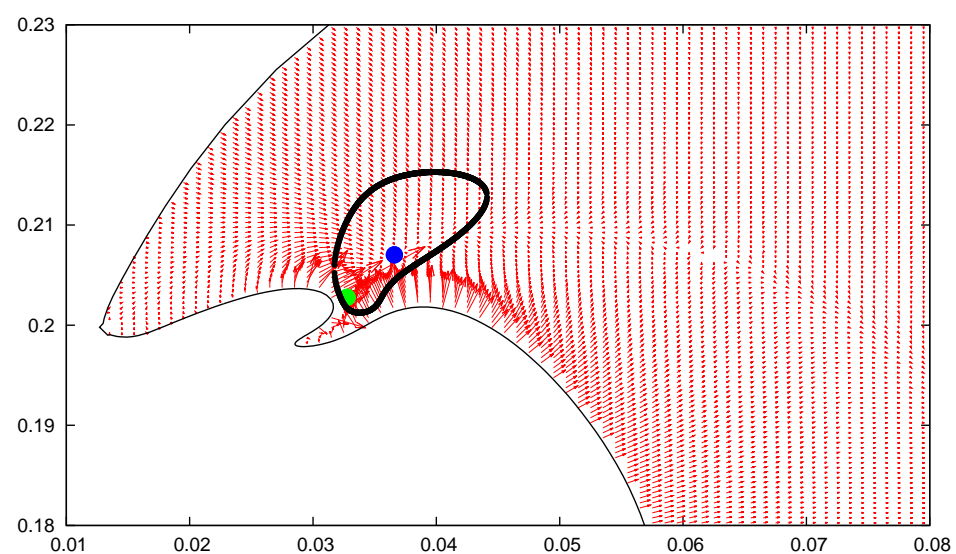

(c)
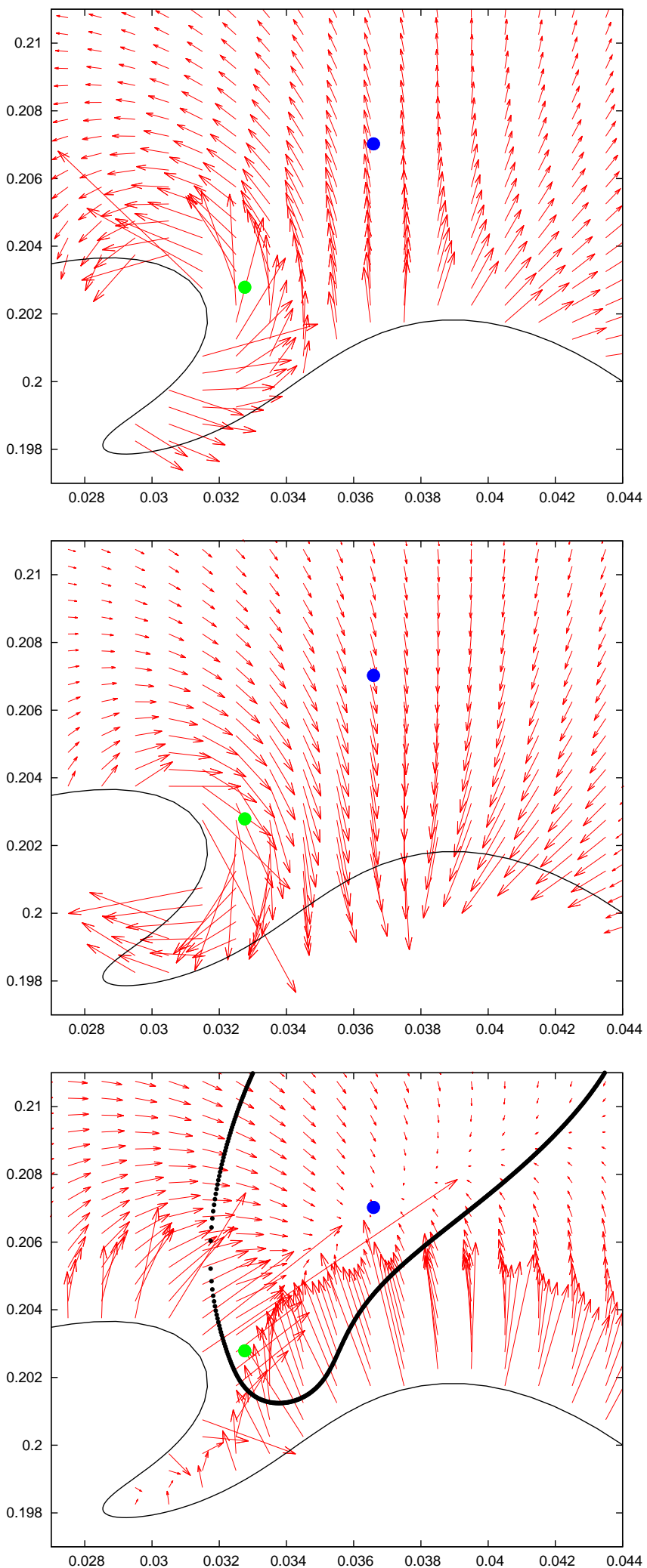

Figure 7: Vector fields of the (a) acceleration $\vec{u}_{, t}$, (b) convective term $(\vec{u} \cdot \vec{\nabla}) \vec{u}$ and (c) pressure gradient $\frac{\vec{\nabla} p}{\rho}$, at instant $t_{2}=0.1542 \mathrm{~s}$. The right figures are closer views of the left figures. The two cartesian components of the vector are divided respectively by $10^{6}, 10^{6}$ and $210^{5}$ for (a), (b) and (c). The units of the vectors are $m^{2} / s^{2}$, Units of the horizontal and vertical coordinates: $m$. The green and blue marks are the locations of the maximum positive value of the Gaussian curvature $\Omega$ and the minimum of $\|\vec{\nabla} p\|$ respectively. The closed curves delimit the inner region where the Gaussian curvature is positive. 


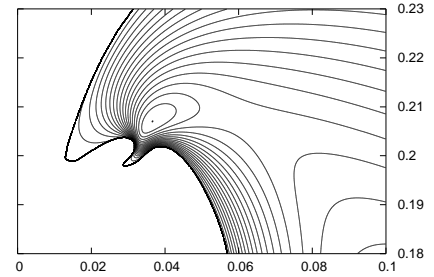

(a)

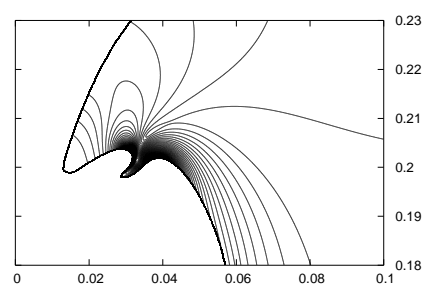

(c)

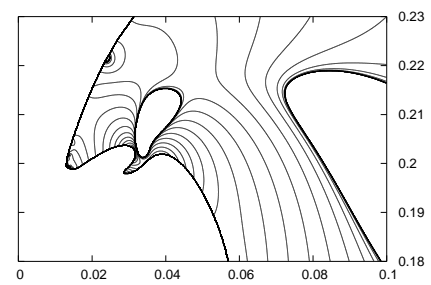

(e)

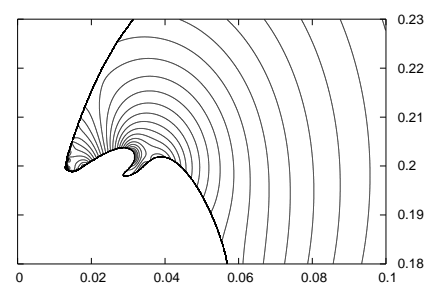

(g)

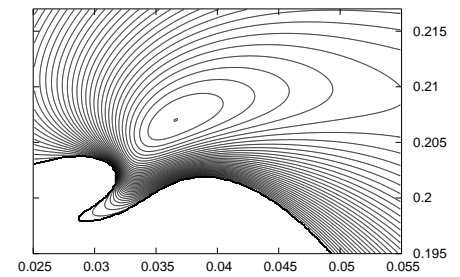

(b)

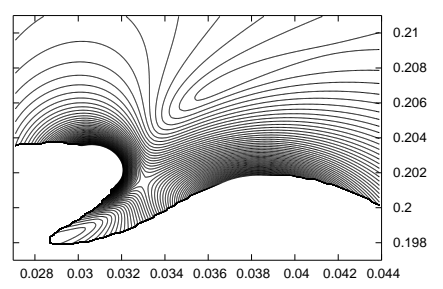

(d)

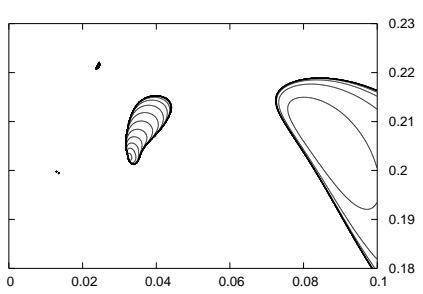

(f)

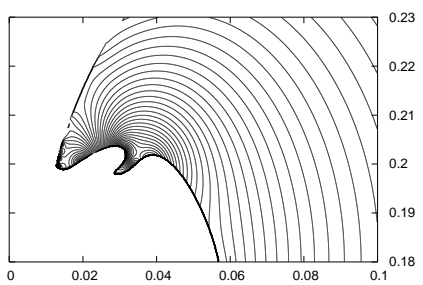

(h)

Figure 8: Isocontours of scalar fields at instant $t_{2}=0.1542 \mathrm{~s}$. The three digits in brackets give the minimum, the step and the maximum of each isocontour plot. (a), (b) : pressure, $(0,0.1,1.95),(0,0.05,1.95)(\mathrm{c}),(\mathrm{d})$ : modulus of the pressure gradient, $(0,20,1800),(0,20,1000),(\mathrm{e}),(\mathrm{f})$ : respectively negative and positive Gaussian curvature, $(-20,0.5,0),(0,0.5,15)(\mathrm{g}): \log$ of the modulus of the acceleration, $(0,0.1,4),(\mathrm{h}): \log$ of the mean curvature. $(0,0.1,10)$, Units of the horizontal and vertical coordinates: $m$. 
(a)
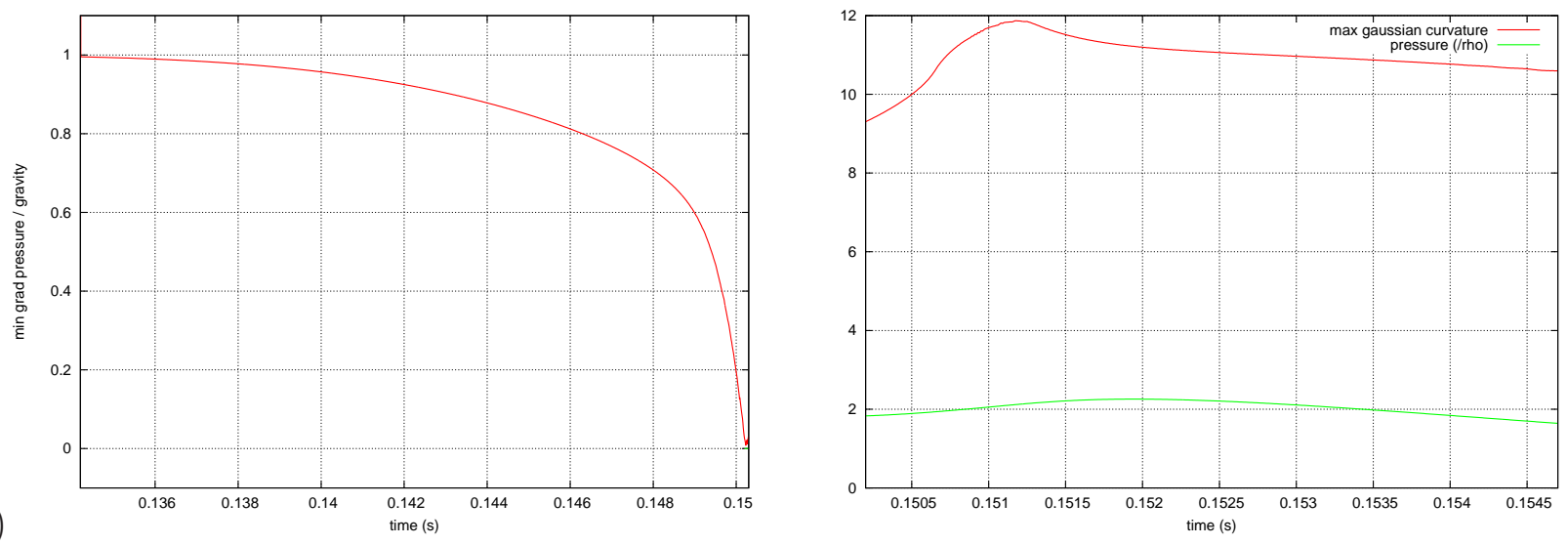

Figure 9: $(a)$ : Time variation of $\frac{1}{g}\|\vec{\nabla} p\|_{\text {min }}$ over the entire simulation. The $(b):$ Time variations of $p_{\max } / \rho$ and $\log _{10} \Omega_{\max }$ since the appearance of the critical jet at time $t \approx 0.15 \mathrm{~s}$, Units: $\mathrm{m}^{2} / \mathrm{s}^{2}$ and $\mathrm{s}^{-2}$

(a)

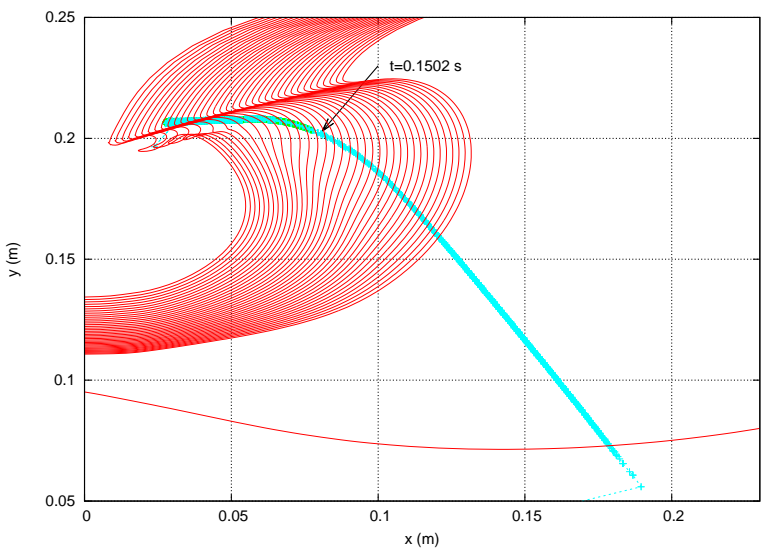

(b)

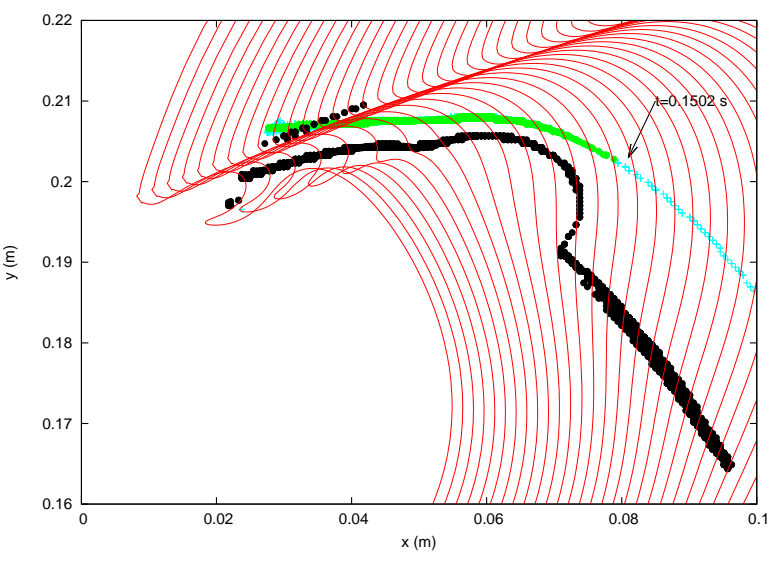

Figure 10: (a) Time tracking of the positions of: Black mark: maximum positive Gaussian curvature. Green mark: maximum pressure. Light blue mark: minimum modulus of the pressure gradient. The arrow indicates the time instant $t=0.15 \mathrm{~s}$ when a local maximum of the pressure appears. (b) Closer view on the tracking after the instant $t=0.15 \mathrm{~s}$, the successive profiles of the free surface are superimposed. Time interval between each profile: $410^{-4} s$. Units of the horizontal and vertical coordinates: $m$. 


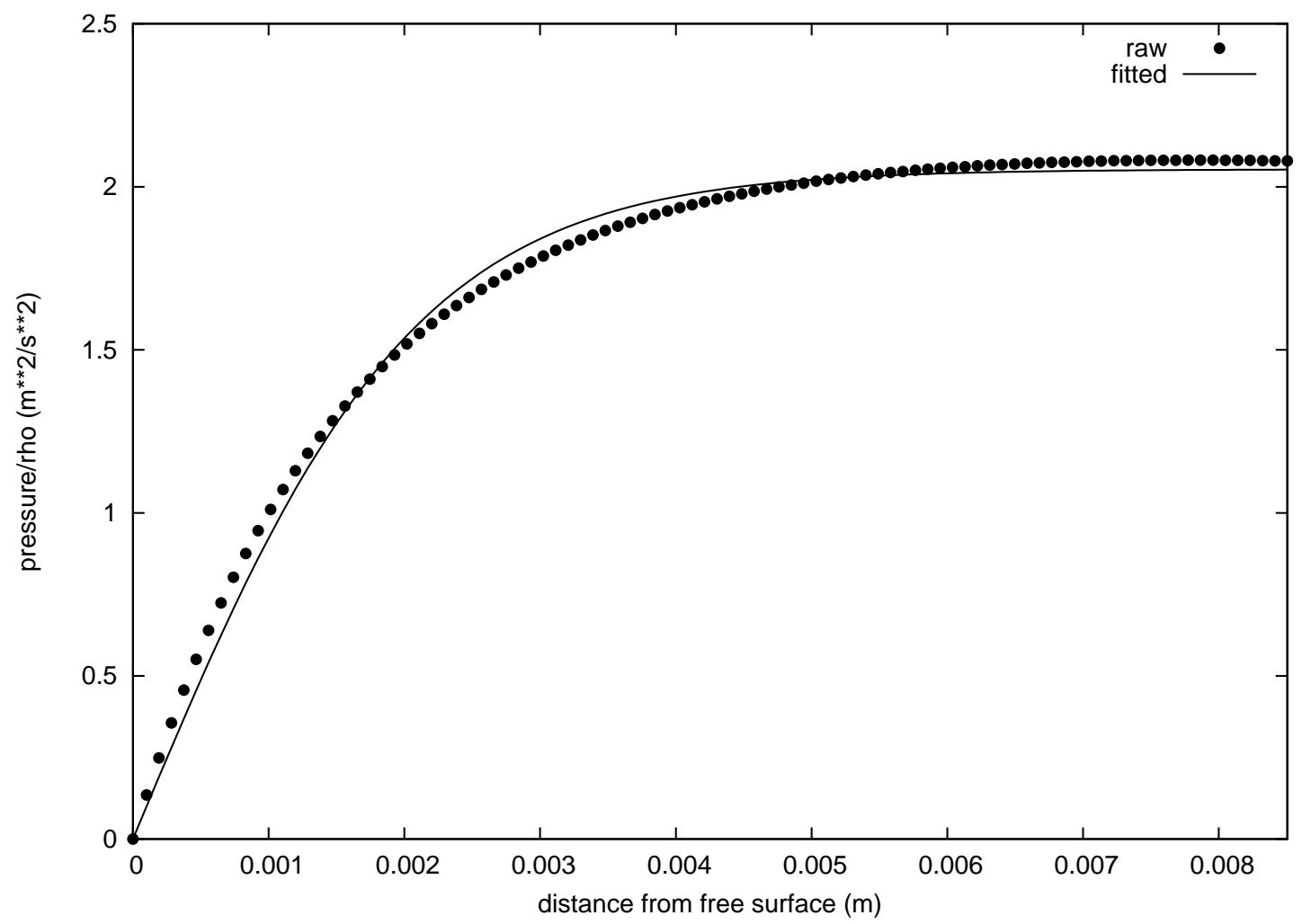

Figure 11: Fitting of a hyperbolic tangent function $p(\sigma) / \rho=\alpha \tanh (\beta \sigma)$ to the variation of the pressure along a straight line joining the two points located at $p_{\max } / \rho$ and $\Omega_{\max } . \sigma$ is the distance from the free surface, $\alpha=2.05 m^{2} / s^{2}$ and $\beta=484 m^{-1}$. 


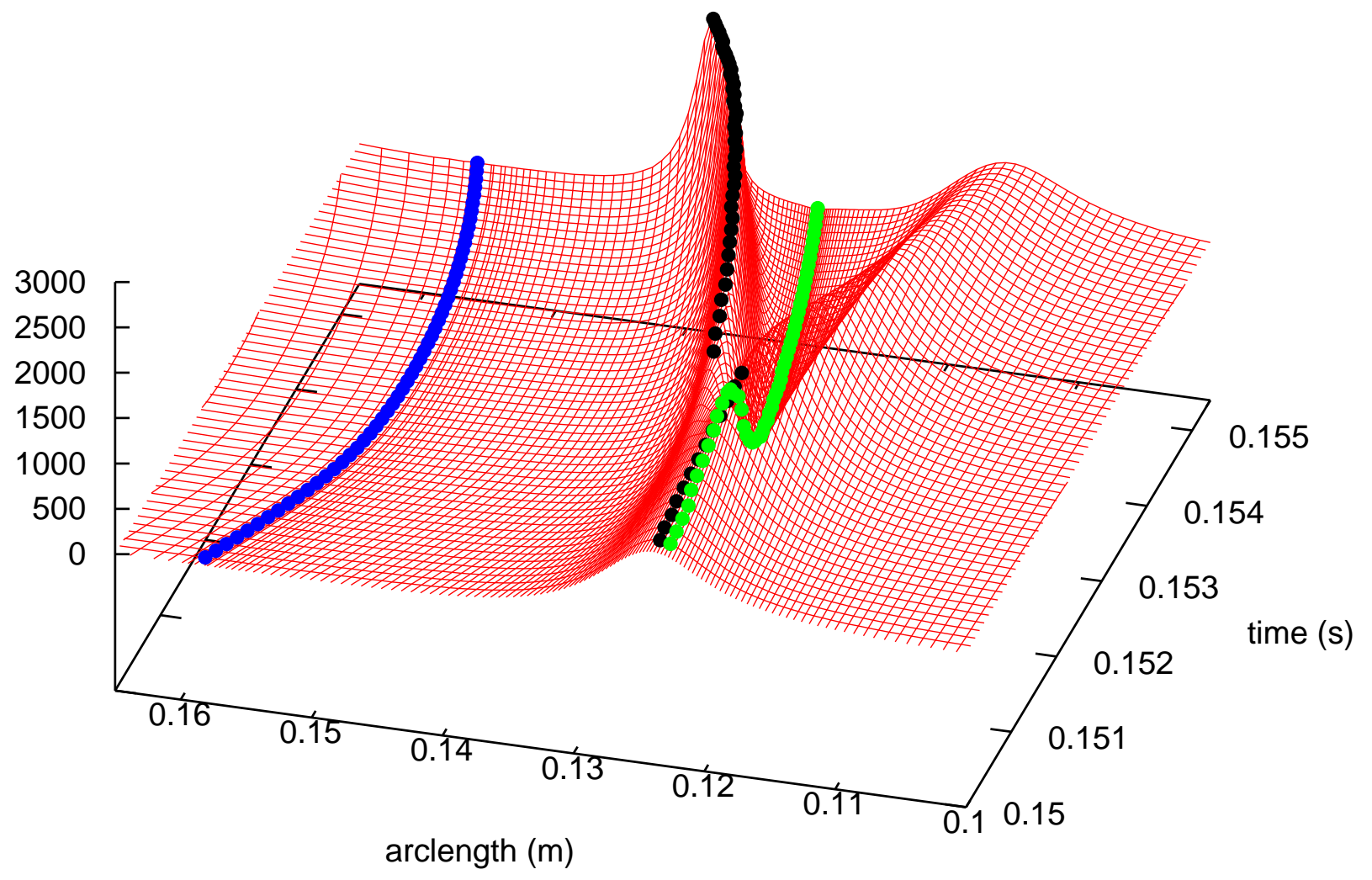

Figure 12: Spatial and temporal variation of the Lagrangian acceleration (amplitude in $\mathrm{m} / \mathrm{s}^{2}$ ) in the time interval $t \in[0.15 \mathrm{~s}, 0.1555 \mathrm{~s}]$. The blue dots follow the main crest. The green dots follow the maximum velocity. The black dots follow the maximum acceleration. Arclength is measured along the free surface from the left wall to the right wall. 


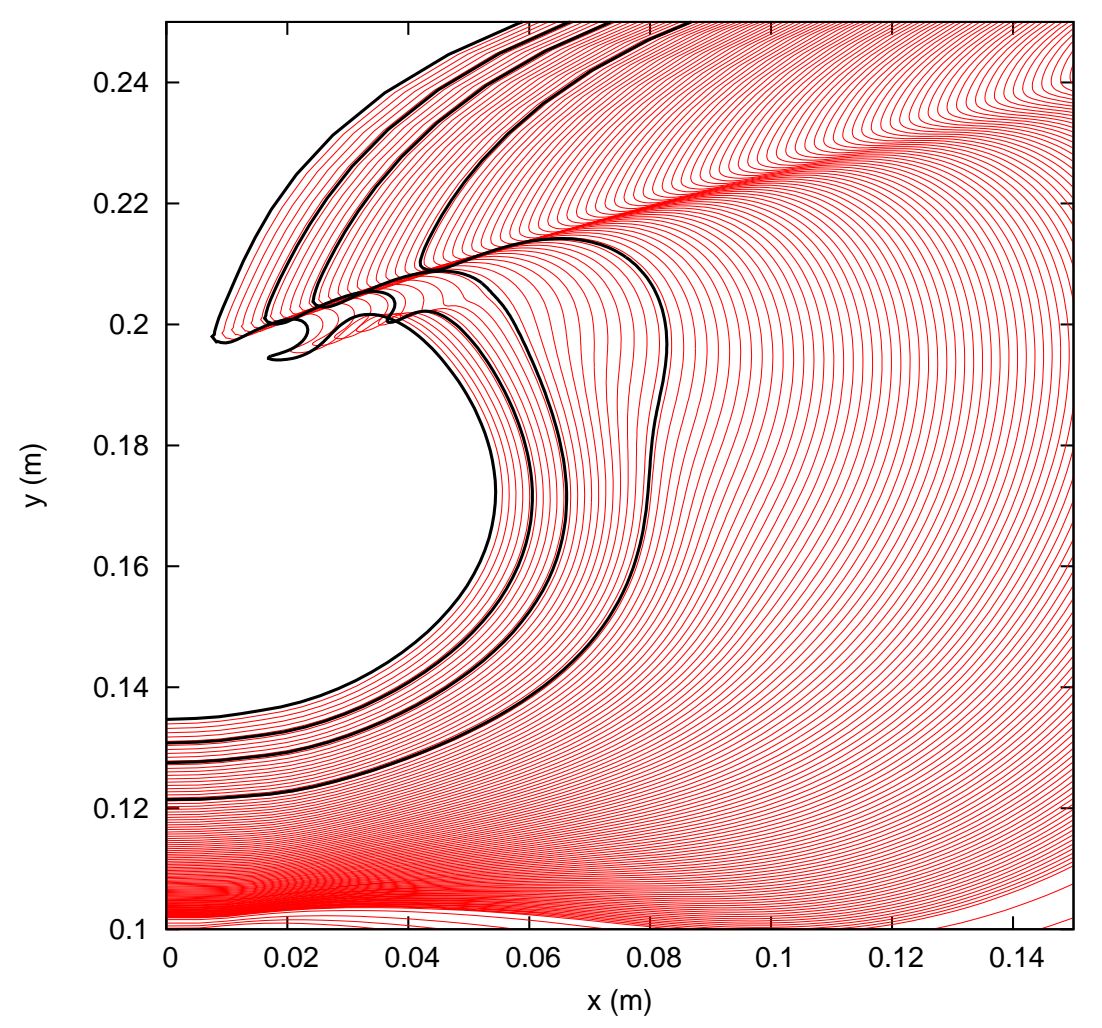

(a)

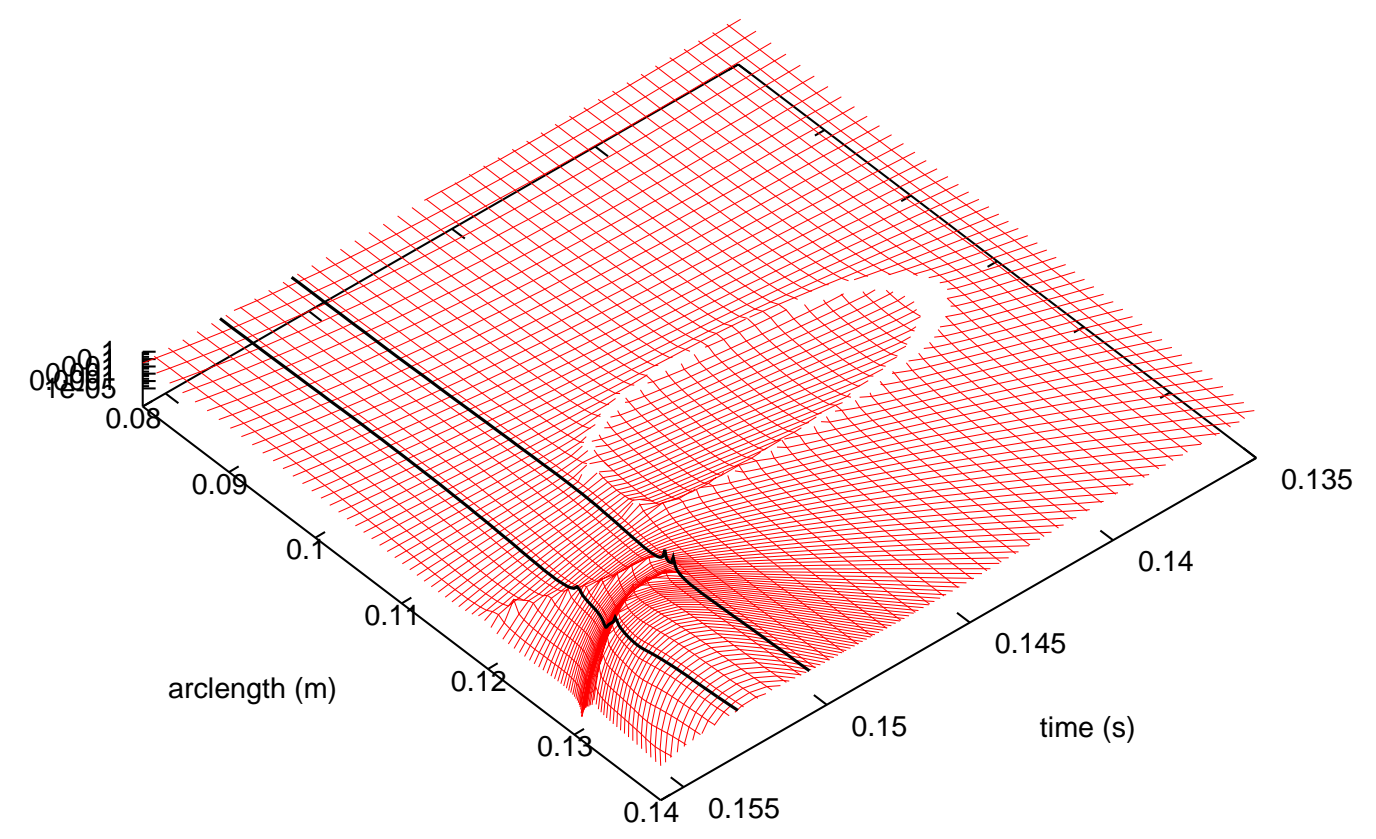

(b)

Figure 13: Restart with $t_{o}=1.63 \mathrm{~s}$. (a) Free surface profiles in the plunging jet. The superimposed black curves correspond to the instants $t=0.1450 \mathrm{~s}, t=0.1506 \mathrm{~s}, t=0.1531 \mathrm{~s}$ and $t=0.1558 \mathrm{~s}$. (b) Spatial and temporal variation of the curvature radius in the time interval $t \in[0.135 \mathrm{~s}, 0.1555 \mathrm{~s}]$. The superimposed black curves correspond to the instants $t=0.1506 \mathrm{~s}$ and $t=0.1531 \mathrm{~s}$. 


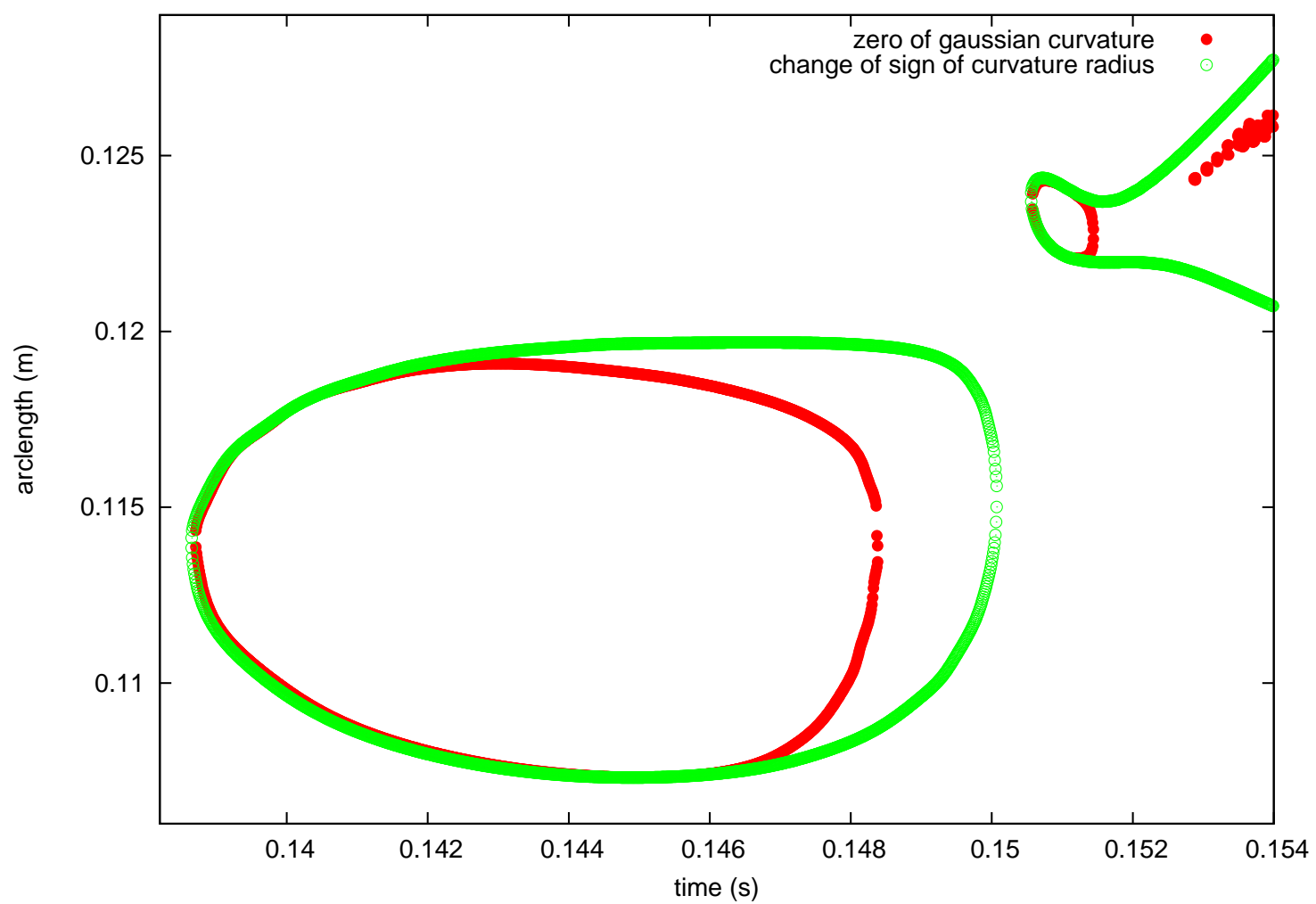

Figure 14: Temporal-spatial variations of the locations where the Gaussian curvature $\Omega$ and radius of cuurvature $R_{c}$ along the free surface change sign. The spatial variation is given by the arclength $\sigma$ measured from the left wall. 